\title{
DISTURBANCE MODELING AND STATE ESTIMATION FOR OFFSET-FREE PREDICTIVE CONTROL WITH STATE-SPACE PROCESS MODELS
}

\author{
PIOTR TATJEWSKI
}

Institute of Control and Computation Engineering

Warsaw University of Technology, ul. Nowowiejska 15/19, 00-665 Warsaw, Poland

e-mail: P.Tatjewski@ia.pw.edu.pl

\begin{abstract}
Disturbance modeling and design of state estimators for offset-free Model Predictive Control (MPC) with linear state-space process models is considered in the paper for deterministic constant-type external and internal disturbances (modeling errors). The application and importance of constant state disturbance prediction in the state-space MPC controller design is presented. In the case with a measured state, this leads to the control structure without disturbance state observers. In the case with an unmeasured state, a new, simpler MPC controller-observer structure is proposed, with observation of a pure process state only. The structure is not only simpler, but also with less restrictive applicability conditions than the conventional approach with extended process-and-disturbances state estimation. Theoretical analysis of the proposed structure is provided. The design approach is also applied to the case with an augmented state-space model in complete velocity form. The results are illustrated on a $2 \times 2$ example process problem.
\end{abstract}

Keywords: model predictive control, state-space models, disturbance rejection, state observer, Kalman filter.

\section{Introduction}

The aim of this paper is twofold: first, to present a systematic explanation of the way the deterministic constant-type disturbances entering the process at any point, possibly with white noise added, can be effectively treated to obtain offset-free model predictive control, under (asymptotically) constant set-point values. The examined class of disturbances, important particularly in process control, includes modeling errors and outer step or piecewise-constant disturbances changing rarely (with respect to controlled process dynamics). Second, the closely related problem of state observer (or Kalman filter) design, when operating with MPC algorithms for processes under disturbances from the stated class, will be presented. Linear state-space process models will be considered in the paper, for which new and competitive control structures will be proposed.

Among the advanced control techniques (see, e.g., Blevins et al., 2003; 2013; Tatjewski, 2007). Model Predictive Control (MPC) is now a well established general technology, resulting in a variety of very successful control techniques applied in practice (see, e.g., Camacho and Bordons, 1999; Maciejowski, 2002; Qin and Badgwell, 2003; Rossiter, 2003; Blevins et al.,
2003; 2013; Tatjewski, 2007; 2008; 2010; Wang, 2009; Rao and Rawlings, 2009). While nonparametric models (like step responses) and transfer function models lead to well established MPC structures, as DMC and GPC, respectively, state-space modeling results in a variety of possibilities.

Different approaches to state-space modeling (as minimal and non-minimal models with full measured state, models with state observers, extended velocity form state-space models including integrators) lead to different handling of deterministic disturbances. Another important factor are points and ways these disturbances influence the process. The mentioned problem had attracted rather limited attention in the literature until the last decade (Muske and Badgwell, 2002; Pannocchia and Rawlings, 2003; Tatjewski, 2007; 2011; Pannocchia and Bemporad, 2007; Gonzalez et al., 2008; Maeder and Morari, 2010; Morari and Maeder, 2012). However, there can still be seen a certain lack of clear understanding how disturbances should be most effectively treated in appropriate MPC algorithms with state-space models. A closely related problem is the way that state observers or Kalman filters should be designed within MPC control structures, as this occurs not to be unique even in general terms (which variables to estimate), and also not 
sufficiently well understood and explained in the current literature. We concentrate on these problems in the paper, for the class of deterministic disturbances defined above. More general, continuously varying disturbances, like sinusoids, are not considered (see the work of Maeder and Morari (2010) for offset-free MPC reference tracking under varying disturbances). It is hard to say, at the moment, whether it is possible to generalize the approaches proposed in the paper to this case.

The structure of the paper is as follows. First, in Section 2, MPC is briefly recalled, to introduce formulations needed for further discussion. In Section 3, the case with a state-space process model assuming full state measurement is considered, with disturbances entering the process input or directly the process states (thus, including also modeling errors). It is explained that, when the constant state disturbance prediction is used (Tatjewski, 2007), then the observer for deterministic disturbances considered is not needed. In Section 4, the case with an unmeasured state and state observers is described. It is argued that the standard approach of extension of the process dynamics by states of deterministic disturbances and then estimation of the extended process-and-disturbance state (see Muske and Badgwell, 2002; Maciejowski, 2002; Pannocchia and Bemporad, 2007; Gonzalez et al., 2008; Morari and Maeder, 2012), although correct, may not be the best solution. Using, appropriately, a pure process state observer only is proposed as more efficient for the class of disturbances considered. It is shown that then the observer estimates not exactly the actual process state, but the state shifted by appropriate values corresponding to disturbances. It is proved that the use of constant state disturbance prediction and an appropriate correction term in output prediction equations provides offset-free control. In Section 5, the case of a non-standard, extended process model in complete velocity form (incremental process state vector augmented by the outputs) is considered (Prett and Garcia, 1988; Gonzalez et al., 2008). It is shown that also in this case the simpler original process state observer followed by appropriate recalculations seems to be a sound solution. The proposed design methods are illustrated on a multivariable $(2 \times 2)$ example process model representing a linearized continuous flow reactor.

\section{Predictive control briefly recalled}

The principle and various algorithms of MPC are presented in many papers and books, including those with discrete-time state-space process models we are interested in (see, e.g., Maciejowski, 2002; Rossiter, 2003; Tatjewski, 2007; Wang, 2009). Therefore, we shall only briefly recall what will be needed for further presentation.

The principle of MPC is to evaluate the current control signal by minimizing, at each sampling instant $k$, a performance function (cost function) on the future prediction horizon of $N$ samples. The following performance function (with $\operatorname{dim} y=\operatorname{dim} u$ assumed, for simplicity of presentation) is one of the most widely used:

$$
\begin{aligned}
J(k)= & \sum_{p=1}^{N} \|\left[y^{s p}(k+p \mid k)-y(k+p \mid k) \|_{\Psi}^{2}\right. \\
& +\sum_{p=0}^{N_{u}-1}\|\Delta u(k+p \mid k)\|_{\Lambda}^{2},
\end{aligned}
$$

where $\|x\|_{\mathbf{R}}^{2}=x^{T} \mathbf{R} x, \boldsymbol{\Psi} \geq \mathbf{0}$ and $\boldsymbol{\Lambda}>\mathbf{0}$ are square diagonal scaling matrices of dimensions corresponding to those of the process controlled output and control input vectors, $\operatorname{dim} y=n_{y}$ and $\operatorname{dim} u=n_{u}$ (often simpler formulation of (1) is used in basic theoretical deliberations, with one scaling scalar $\lambda$ only, i.e., $\Psi=\mathbf{I}$ and $\boldsymbol{\Lambda}=\lambda \mathbf{I})$. In the above, $N_{u} \leq N$ denotes the length of the control horizon, $y^{s p}(k+p \mid k)$ and $y(k+p \mid k)$ are set-points (reference values) and outputs predicted for a future sample $k+p$, but calculated at the current sample $k, p=1, \ldots, N$. The decision variables are control increments on the control horizon, $\Delta u(k+p \mid k)=$ $u(k+p \mid k)-u(k+p-1 \mid k), p=0, \ldots, N_{u}-1$.

The optimization of $J(k)$ is performed subject to constraints (a more general form of constraints, including any linear functions of all variables used, is possible, but avoided here for presentation simplicity):

$$
\begin{aligned}
& -\Delta u_{\max } \leq \Delta u(k+p \mid k) \leq \Delta u_{\max }, \\
& \quad p=0, \ldots, N_{u}-1, \\
& u_{\min } \leq u(k+p \mid k) \leq u_{\max }, \quad p=0, \ldots, N_{u}-1, \\
& y_{\min } \leq y(k+p \mid k) \leq y_{\max }, \quad p=1, \ldots, N .
\end{aligned}
$$

The superposition principle can be applied when using linear process models; then the predicted trajectory of the outputs can be split into the "free trajectory" $\left\{y^{0}(k+p \mid k), p=1, \ldots, N\right\}$, depending on current and past data only (obtained with the control frozen over the prediction horizon on the last applied value $u(k-1)$ ) and the "forced trajectory" $\left\{y^{+}(k+p \mid k), p=1, \ldots, N\right\}$, depending on the vector of decision variables only,

$$
\begin{aligned}
y(k+p \mid k)=y^{0}(k+p \mid k)+y^{+}(k+p \mid k), & \\
& p=1, \ldots, N .
\end{aligned}
$$

Denoting the vector of decision variables by $\Delta U(k)$,

$$
\begin{aligned}
\Delta U(k)=\left[\Delta u(k \mid k)^{T} \Delta u(k+1 \mid k)^{T} \cdots\right. \\
\left.\cdots \Delta u\left(k+N_{u}-1 \mid k\right)^{T}\right]^{T},
\end{aligned}
$$

we have, due to linearity of the process model,

$$
y^{+}(k+p \mid k)=\mathbf{M}_{p} \Delta U(k), \quad p=1, \ldots, N,
$$


where the matrix $\mathbf{M}=\left[\mathbf{M}_{1}^{T} \cdots \mathbf{M}_{N}^{T}\right]^{T}$ consists of process step response coefficients (it is often called the dynamic matrix).

Denote

$$
\begin{gathered}
Y^{s p}(k)=\left[\begin{array}{lll}
y^{s p}(k+1 \mid k)^{T} & \cdots & y^{s p}(k+N \mid k)^{T}
\end{array}\right]^{T}, \\
Y^{0}(k)=\left[\begin{array}{lll}
y^{0}(k+1 \mid k)^{T} & \cdots & y^{0}(k+N \mid k)^{T}
\end{array}\right]^{T}, \\
Y^{+}(k)=\left[\begin{array}{lll}
y^{+}(k+1 \mid k)^{T} & \cdots & y^{+}(k+N \mid k)^{T}
\end{array}\right]^{T}, \\
\underline{\boldsymbol{\Psi}}=\operatorname{diag}\{\overbrace{\boldsymbol{\Psi}, \ldots, \boldsymbol{\Psi}}^{N \text { times }}\}, \quad \underline{\boldsymbol{\Lambda}}=\operatorname{diag}\{\overbrace{\boldsymbol{\Lambda}, \ldots, \boldsymbol{\Lambda}}^{N_{u} \text { times }}\} .
\end{gathered}
$$

Then we can formulate in compact form the way the MPC algorithm calculates the control trajectory. It is the following MPC optimization problem:

$$
\begin{aligned}
\min _{\Delta U(k)}\{J(k)= & \|\left[Y^{s p}(k)-Y^{0}(k)-\mathbf{M} \Delta U(k) \|_{\underline{\underline{\Psi}}}^{2}\right. \\
& \left.+\|\Delta U(k)\|_{\underline{\Lambda}}^{2}\right\}
\end{aligned}
$$

subject to (2)-(4).

Under the stated assumptions, the MPC optimization problem (12) is a strictly convex Quadratic Programming (QP) problem, thus with a well-defined, unique solution, provided the set defined by the constraints assures feasibility (is non-empty).

All the formulae given in this section are general, valid for linear process models in any form-for different kinds of models different formulae for the matrix $\mathbf{M}$ and the predicted output free trajectory $Y^{0}(k)$ result.

\section{MPC with a state-space model and a measured state}

We shall describe MPC controllers with state-space process models as MPCS (MPC with a State-space model) controllers. Let us assume now the following state-space process model:

$$
\begin{aligned}
x(k+1) & =\mathbf{A} x(k)+\mathbf{B} u(k)+v(k), \\
y(k) & =\mathbf{C} x(k),
\end{aligned}
$$

where $x$ is the state vector of dimension $n_{x}$, assumed measured in this section, $y$ is the output vector of dimension $n_{y}$ and $v$ is the state disturbance vector, representing constant-type disturbances of the class considered. Using this model, recursively, we can easily obtain the formulae for $Y^{0}(k)$ and $\mathbf{M}$ (see Tatjewski, 2007),

$$
Y^{0}(k)=\widetilde{\mathbf{C}} \widetilde{\mathbf{A}} x(k)+\widetilde{\mathbf{C}} \mathbf{V B} u(k-1)+\widetilde{\mathbf{C}} \mathbf{V} v(k),
$$

$$
\mathbf{M}=\left[\begin{array}{ccc}
\mathbf{C B} & \cdots & \mathbf{0} \\
\mathbf{C}(\mathbf{I}+\mathbf{A}) \mathbf{B} & \cdots & \mathbf{0} \\
\mathbf{C}\left(\mathbf{I}+\mathbf{A}+\mathbf{A}^{2}\right) \mathbf{B} & \cdots & \mathbf{0} \\
\vdots & \ddots & \vdots \\
\mathbf{C}\left(\mathbf{I}+\cdots+\mathbf{A}^{N_{u}-1}\right) \mathbf{B} & \cdots & \mathbf{C B} \\
\mathbf{C}\left(\mathbf{I}+\cdots+\mathbf{A}^{N_{u}}\right) \mathbf{B} & \cdots & \mathbf{C}(\mathbf{I}+\mathbf{A}) \mathbf{B} \\
\vdots & \vdots & \vdots \\
\mathbf{C}\left(\mathbf{I}+\cdots+\mathbf{A}^{N-1}\right) \mathbf{B} & \cdots & \mathbf{C}\left(\mathbf{I}+\cdots+\mathbf{A}^{N-N_{u}}\right) \mathbf{B}
\end{array}\right]
$$

where

$$
\begin{gathered}
\widetilde{\mathbf{C}}=\operatorname{diag}\{\overbrace{\mathbf{C}, \cdots, \mathbf{C}}^{N \text { times }}\} \\
\widetilde{\mathbf{A}}=\left[\begin{array}{c}
\mathbf{A} \\
\mathbf{A}^{2} \\
\mathbf{A}^{3} \\
\vdots \\
\mathbf{A}^{N}
\end{array}\right], \quad \mathbf{V}=\left[\begin{array}{c}
\mathbf{I} \\
\mathbf{A}+\mathbf{I} \\
\mathbf{A}^{2}+\mathbf{A}+\mathbf{I} \\
\vdots \\
\mathbf{A}^{N-1}+\cdots+\mathbf{A}+\mathbf{I}
\end{array}\right]
\end{gathered}
$$

To predict $v(k)$, the constant state disturbance prediction model was proposed by Tatjewski (2007):

$$
\begin{aligned}
v(k) & =x(k)-[\mathbf{A} x(k-1)+\mathbf{B} u(k-1)], \\
v(k+1 \mid p) & =v(k+2 \mid p)=\cdots v(k+N-1 \mid k)=v(k) .
\end{aligned}
$$

The information given above is sufficient for the formulation and solution of the QP problem of the state-space model based MPC (MPCS) algorithm. The above formulation of the MPCS algorithm with state disturbances $v(k)$ and their prediction (19) used in (15) assures offset-free disturbance attenuation for any process input and state disturbances of the class considered.

Process outputs are usually certain elements of the state vector or linear combinations of its elements; then the output equation is a precise one, not introducing any output disturbances (state is measured), and the approach presented above is sufficient, complete. However, there are cases when the original output equation is nonlinear, $y(k)=g(x(k))$, as, e.g., in the case of the polymerization reactor considered by Doyle III et al. (1996), where the output is a nonlinear function of the process states. Then, using a linearized output equation results in an output disturbance of modeling error type, which cannot be attenuated in the structure considered with a measured state and state disturbances $v(k)$. That is because the feedback information used in the MPCS algorithm as "output measurement" is $\mathbf{C} x(k)$ - the true nonlinear output $g\left(x(k)+x_{0}\right)$ is outside the feedback loop (where $x_{0}$ is the working point at which the linearized state-space model has been derived and is applied). A straightforward analytical compensation, based on the knowledge of the nonlinear output equation, is recommended here (see Tatjewski, 2007). Technically, it can be treated as "output 
disturbance" $d(k)$,

$$
d(k)=g\left(x(k)+x_{0}\right)-\left[g\left(x_{0}\right)+\mathbf{C} x(k)\right] .
$$

It is calculated and added to the predicted output free trajectory (15), which then takes the form

$$
\begin{aligned}
Y^{0}(k)= & \widetilde{\mathbf{C}} \widetilde{\mathbf{A}} x(k)+\widetilde{\mathbf{C}} \mathbf{V B} u(k-1) \\
& +\widetilde{\mathbf{C}} \mathbf{V} v(k)+\widetilde{\mathbf{I}}_{y} d(k),
\end{aligned}
$$

where $\widetilde{\mathbf{I}}_{y}=[\overbrace{\mathbf{I}_{y} \mathbf{I}_{y} \cdots \mathbf{I}_{y}}^{N \text { times }}]^{T}, \operatorname{dim} \mathbf{I}_{y}=n_{y} \times n_{y}$.

It should be mentioned that the case with a full state measured is of practical value not only, e.g., in mechatronic applications (like robotics), but also in process control, where the use of non-minimal state representations, with state vectors consisting of current and past process outputs and inputs, introduced originally by Hesketh (1982) within a pole-placement control context, can be applied to avoid the necessity of using state observers or filters (see the works of Maciejowski (2002), Tatjewski (2007) and Wang (2009) for applications in MPC algorithms). In particular, models of dynamic processes in the form of difference equations can be easily transformed to the above-mentioned state-space form. This case includes also neural-network models important for on-line applications of MPC control (Tatjewski and Ławryńczuk, 2006; Ławryńczuk, 2009; Ławryńczuk and Tatjewski, 2010).

Finally, let us point out the rather known fact that, applying mechanically, for constant-type disturbances, the approach copied from classical DMC or GPC algorithms, with constant output disturbance prediction used only,

$$
\begin{aligned}
d(k) & =y(k)-y(k \mid k-1) \\
& =y(k)-\mathbf{C} x(k \mid k-1) \\
& =y(k)-\mathbf{C}[\mathbf{A} x(k-1)+\mathbf{B} u(k-1)],
\end{aligned}
$$

is not correct. It does not usually provide offset-free control, when used without any other estimation of state (or input) disturbances. Therefore, the standard solution was to add a dynamic model of disturbances and next, consequently, an observer. The approach with constant state disturbance prediction 19-20 is a simpler, sound solution, not needing the observer (Tatjewski, 2007). Further, we shall utilize this approach to design a simpler and more general MPC controller-observer control structure, for the case with an unmeasured state.

\section{MPCS with state observation/estimation}

The entire state vector is often not accessible, especially when using minimal state-space representations. State estimation using observers or Kalman filters is then a standard solution. We concentrate on state observers, as the presentation is then simpler and can be easily generalized.

For the process described by (13)-114), the state observer of Luenberger type can be formulated as follows (the predictive observer (see, e.g., Astrom and Wittenmark, 1997)):

$$
\hat{x}(k+1)=\mathbf{A} \hat{x}(k)+\mathbf{B} u(k)+\mathbf{K}_{\mathrm{obs}}[y(k)-\mathbf{C} \hat{x}(k)],
$$

where $\hat{x}(k)$ denotes the state estimate and $\mathbf{K}_{\mathrm{obs}}$ is the gain matrix of the observer. As well known, observability of a dynamic system given by matrices $\mathbf{A}, \mathbf{B}, \mathbf{C}$ makes it possible, by a suitable choice of $\mathbf{K}_{\mathrm{obs}}$, to get any desirable location of poles of the observer state matrix $\mathbf{A}-\mathbf{K}_{\mathrm{obs}} \mathbf{C}$. This matrix describes also the dynamics of the estimation error, $e_{\mathrm{obs}}(k)=x(k)-\hat{x}(k)$, as we can easily get

$$
e_{\mathrm{obs}}(k+1)=\left(\mathbf{A}-\mathbf{K}_{\mathrm{obs}} \mathbf{C}\right) e_{\mathrm{obs}}(k) .
$$

Having the state estimate $\hat{x}(k)$ and inserting it, in place of the state $x(k)$, into 19 ,

$$
v(k)=\hat{x}(k)-[\mathbf{A} \hat{x}(k-1)+\mathbf{B} u(k-1)],
$$

and into 15 , we get the formula for the free predicted output trajectory, corresponding to 15 :

$$
Y^{0}(k)=\widetilde{\mathbf{C}} \widetilde{\mathbf{A}} \hat{x}(k)+\widetilde{\mathbf{C}} \mathbf{V B} u(k-1)+\widetilde{\mathbf{C}} \mathbf{V} v(k)
$$

However, it should be realized that the presented observer estimates the process steady-state correctly only if deterministic disturbances have zero steady-state. Under constant non-zero disturbances, the estimated state will not be equal to the true process state, but will converge to a shifted state value, biased by the disturbances. This follows directly from the disturbed process and observer equations, as presented below.

Consider the process equation with a general state disturbance vector $z(k) \in \mathbb{R}^{n_{z}}, n_{z} \leq n_{x}$,

$$
x(k+1)=\mathbf{A} x(k)+\mathbf{B} u(k)+\mathbf{G} z(k) .
$$

Observe that, when $\mathbf{G}=\mathbf{B}$, then $z(k)$ are pure input disturbances (acting on the process input), when $\mathbf{G}=\mathbf{I}$, $z(k)$ are disturbances affecting directly the next state, while with $\mathbf{G} z(k)=\delta \mathbf{A} x(k)$ we have a representation of modeling errors. Taking now into account the observer and output equations, (24) and (14), we get

$$
e_{\mathrm{obs}}(k+1)=\left(\mathbf{A}-\mathbf{K}_{\mathrm{obs}} \mathbf{C}\right) e_{\mathrm{obs}}(k)+\mathbf{G} z(k) .
$$

Assuming asymptotic stability of the feedback control system, a constant set-point and constant (in steady-state) disturbance value $z_{s s}$, we obtain at the equilibrium (in steady-state) the following constant observation error:

$$
e_{\mathrm{obs}, \mathrm{ss}}=\left(\mathbf{I}-\mathbf{A}+\mathbf{K}_{\mathrm{obs}} \mathbf{C}\right)^{-1} \mathbf{G} z_{s s}
$$


which is not zero for $z_{s s} \neq 0$. Observe that we have at steady-state (at the equilibrium), in general, $\hat{x}_{s s} \neq$ $x_{s s}, y_{s s}=\mathbf{C} x_{s s}$ and a non-zero correction term in the observer equation, $y_{s s}-\mathbf{C} \hat{x}_{s s} \neq 0$ (it may happen that $y_{s s}=\mathbf{C} \hat{x}_{s s}$, occasionally, despite $\left.\hat{x}_{s s} \neq x_{s s}\right)$.

Nevertheless, the presented MPCS controller with the (not augmented) state observer (24) will work correctly, with offset-free control, provided the predicted output free trajectory is extended to the following form:

$$
\begin{aligned}
Y^{0}(k)= & \widetilde{\mathbf{C}} \widetilde{\mathbf{A}} \hat{x}(k)+\widetilde{\mathbf{C}} \mathbf{V B} u(k-1)+\widetilde{\mathbf{C}} \mathbf{V} v(k) \\
& +\widetilde{\mathbf{I}}_{y}[y(k)-\mathbf{C} \hat{x}(k)] .
\end{aligned}
$$

This statement will now be proved.

Consider prediction equations of the state, as used in 31, with the constant state disturbance predictions (26) taken into account, and formulated recursively:

$$
\begin{aligned}
x^{0}(k+1 \mid k)= & \mathbf{A} \hat{x}(k)+\mathbf{B} u(k-1) \\
& +[\hat{x}(k)-\mathbf{A} \hat{x}(k-1)-\mathbf{B} u(k-1)],
\end{aligned}
$$

$$
\begin{aligned}
x^{0}(k+2 \mid k)= & \mathbf{A} x^{0}(k+1 \mid k)+\mathbf{B} u(k-1) \\
& +[\hat{x}(k)-\mathbf{A} \hat{x}(k-1)-\mathbf{B} u(k-1)],
\end{aligned}
$$

$$
\begin{aligned}
x^{0}(k+p \mid k)= & \mathbf{A} x^{0}(k+p-1)+\mathbf{B} u(k-1) \\
& +[\hat{x}(k)-\mathbf{A} \hat{x}(k-1)-\mathbf{B} u(k-1)],
\end{aligned}
$$

and so on, until $p=N$.

Assuming the set-point signal is (asymptotically) constant, $y^{s p}(k)=y^{s p}$, and feasible at steady-state, disturbances are constant and the control system is asymptotically stable, the following conditions hold at steady-state:

- states $x(k)$ and their estimates $\hat{x}(k)$ stabilize at constant values $x_{s s}$ and $\hat{x}_{s s}$,

- the performance function (1) stabilizes at the most minimal, zero value, as the set-point is assumed feasible and control increments are zero at steady-state, which means that

$$
y^{s p}=y^{0}(k+p \mid k), \quad p=1, \ldots, N .
$$

Taking into account the above conditions, without the correction term $y(k)-\mathbf{C} \hat{x}(k)$ the free part (31) of the predictive output trajectory would take, at steady state, the form

$$
\begin{aligned}
y^{0}(k+p \mid k) & =\mathbf{C} x^{0}(k+p \mid k) \\
& =\mathbf{C}\left[\mathbf{A} \hat{x}_{s s}+\left(\hat{x}_{s s}-\mathbf{A} \hat{x}_{s s}\right)\right] \\
& =\mathbf{C} \hat{x}_{s s}, \quad p=1, \ldots, N
\end{aligned}
$$

which can be obtained by examining, recursively, Eqns. (32a)-32c) at steady-state: $\hat{x}(k)$ stabilizes at $\hat{x}_{s s}$; then from (32a) $x^{0}(k+1 \mid k)=\hat{x}_{s s}$, which with 32b implies that $x^{0}(k+2 \mid k)=\hat{x}_{s s}$, etc. until $x^{0}(k+N \mid k)=\hat{x}_{s s}$.

Therefore, 33 implies that the predicted outputs, equal to $\mathbf{C} \hat{x}_{s s}$, would stabilize at the set-point value $y^{s p}$. But, adding to every output prediction equation the correction term $y(k)-\mathbf{C} \hat{x}(k)$, see (31), which at steady-state is equal to $y_{s s}-\mathbf{C} \hat{x}_{s s}$, shifts the predicted output value $\mathbf{C} \hat{x}_{s s}$ to the real output value $y_{s s}$; therefore at steady-state we get the offset-free stabilization:

$$
y^{s p}=y^{0}(k+p \mid k)=\mathbf{C} \hat{x}_{s s}+\left[y_{s s}-\mathbf{C} \hat{x}_{s s}\right]=y_{s s},
$$

which completes the proof.

The described technique works properly due to two factors: the first and key factor is the proper state prediction due to the use of the constant state disturbance predictions 26, the second one is the use of the appropriate correction term $y(k)-\mathbf{C} \hat{x}(k)$, added to the predicted outputs (after predictions), see (31). It should be noticed that also output disturbances, either representing modeling errors of the output equations or outer influences on the process outputs, will be attenuated, due to the use of the correction term.

The presented technique is not a known, standard one. The standard one applied up to now (see Muske and Badgwell, 2002; Pannocchia and Rawlings, 2003; Maciejowski, 2002; Pannocchia and Bemporad, 2007; Gonzalez et al., 2008; Rao and Rawlings, 2009; Maeder and Morari, 2010) is to augment the process state by the states of deterministic disturbances, which results in the following extended state and output equations:

$$
\begin{aligned}
{\left[\begin{array}{c}
x(k+1) \\
z(k+1) \\
d(k+1)
\end{array}\right] } & =\left[\begin{array}{ccc}
\mathbf{A} & \mathbf{G} & \mathbf{0} \\
\mathbf{0} & \mathbf{I} & \mathbf{0} \\
\mathbf{0} & \mathbf{0} & \mathbf{I}
\end{array}\right]\left[\begin{array}{l}
x(k) \\
z(k) \\
d(k)
\end{array}\right]+\left[\begin{array}{c}
\mathbf{B} \\
\mathbf{0} \\
\mathbf{0}
\end{array}\right] u(k), \\
y(k) & =\left[\begin{array}{lll}
\mathbf{C} & \mathbf{0} & \mathbf{G}_{d}
\end{array}\right]\left[\begin{array}{c}
x(k) \\
z(k) \\
d(k)
\end{array}\right]
\end{aligned}
$$

Then a standard observer is used for estimation of the extended state vector consisting of process and disturbance states, $(x(k), z(k), d(k))$, provided the extended system is detectable. Necessary and sufficient conditions for that are the detectability of $(\mathbf{C}, \mathbf{A})$ and the following rank requirement (Muske and Badgwell, 2002):

$$
\begin{aligned}
\operatorname{rank}\left[\begin{array}{ccc}
\mathbf{I}-\mathbf{A} & -\mathbf{G} & \mathbf{0} \\
\mathbf{C} & \mathbf{0} & \mathbf{G}_{d}
\end{array}\right] \\
\quad=\operatorname{dim} x+\operatorname{dim} z+\operatorname{dim} d .
\end{aligned}
$$

In particular, assuming full column ranks of $\mathbf{G}$ and $\mathbf{G}_{d}$, the condition (38) implies that the extended model is not detectable if $\operatorname{dim} z+\operatorname{dim} d>\operatorname{dim} y$, which may be restrictive. 
Applying the conventional approach presented above, we should apply the following formula for predicted output free trajectory $Y^{0}(k)$ (instead of (31)):

$$
\begin{aligned}
Y^{0}(k)= & \widetilde{\mathbf{C}} \widetilde{\mathbf{A}} \hat{x}(k)+\widetilde{\mathbf{C}} \mathbf{V}(\mathbf{B} u(k-1) \\
& +\mathbf{G} \hat{z}(k))+\widetilde{\mathbf{I}}_{y} \hat{d}(k),
\end{aligned}
$$

where $\hat{z}(k), \hat{d}(k)$ are estimates of disturbances obtained from the observer of the augmented state.

The MPC controller-observer structure proposed in the paper, with the constant state disturbance prediction (26), is a sound alternative to the conventional approach just described above. It should be pointed out that the proposed approach is simpler and operates under the pure process detectability condition only (pair $(\mathbf{A}, \mathbf{C})$ detectable), not imposing additional conditions. On the other hand, the requirement of detectability of the extended system 36-37) in the conventional approach implies the additional condition (38), limiting the number of deterministic disturbances by that of measured outputs $(\operatorname{dim} y)$.

The presented reasoning for the system (13)-14) has been so far for the predictive observer (24). A slightly different and popular observer form, known as the current observer (see, e.g., Astrom and Wittenmark, 1997), is given by the equation

$$
\begin{aligned}
\hat{x}(k)= & \mathbf{A} \hat{x}(k-1)+\mathbf{B} u(k-1)+\mathbf{K}_{\mathrm{obs}}[y(k) \\
& -\mathbf{C}(\mathbf{A} \hat{x}(k-1)+\mathbf{B} \hat{u}(k-1))] .
\end{aligned}
$$

The results described for the predictive observer are all true for the current observer (can be similarly obtained).

The reasoning presented so far in this paper was made under the assumption of zero stochastic disturbances (noises). In general, additional state and output noises cannot be neglected, thus, instead of (13) - 114), the system model would be

$$
\begin{aligned}
x(k+1) & =\mathbf{A} x(k)+\mathbf{B} u(k)+v(k)+w_{x}(k), \\
y(k) & =\mathbf{C} x(k)+w_{y}(k),
\end{aligned}
$$

where $w_{x}$ and $w_{y}$ are state and measurement noises, assumed usually to be white Gaussian with zero mean values and covariance matrices $\mathbf{Q}$ and $\mathbf{R}$, respectively. In this case, the use of a Kalman filter instead of a deterministic observer is recommended. The presented results can be easily generalized to this case, as the form of the Kalman filter dynamic equation is identical with that of Luenberger's current observer (40) (Anderson and Moore, 2005; Astrom and Wittenmark, 1997). We have only the Kalman filter gain matrix $\mathbf{K}$, obtained in a different way, instead of the observer gain matrix $\mathbf{K}_{\text {obs }}$. For the stationary (steady-state) Kalman filter, the generalization is straightforward, as its gain matrix $\mathbf{K}$ is then also constant, given by the equation, (see, e.g., Anderson and Moore, 2005)

$$
\mathbf{K}=\mathbf{P C}^{T}\left(\mathbf{C P C} \mathbf{C}^{T}+\mathbf{R}\right)^{-1}
$$

where matrix $\mathbf{P}$ is a solution to the Riccati equation

$$
\mathbf{P}=\mathbf{A}\left[\mathbf{P}-\mathbf{P C}^{T}\left(\mathbf{C P C} \mathbf{C}^{T}+\mathbf{R}\right)^{-1} \mathbf{C P}\right] \mathbf{A}+\mathbf{Q} .
$$

Certainly, the results concerning state estimates and zeroing control errors apply now in the stochastic sense. The proposed MPC control structure, with process state estimation only, works also with a general (not stationary) Kalman filter with a variable gain matrix $\mathbf{K}=\mathbf{K}(k)$ (see Tatjewski, 2012).

Example 1. The following MIMO $(2 \times 2)$ discrete process model, representing linearized (at an operating point) model of a continuous flow reactor will be considered (Camacho and Bordons, 1999; Tatjewski, 2007):

$$
\begin{aligned}
& {\left[\begin{array}{l}
y_{1}(k) \\
y_{2}(k)
\end{array}\right]} \\
& \quad=\left[\begin{array}{ll}
\frac{0.041951 z^{-1}}{1-0.958048 z^{-1}} & \frac{0.475812 z^{-1}}{1-0.904837 z^{-1}} \\
\frac{0.058235 z^{-1}}{1-0.941764 z^{-1}} & \frac{0.144513 z^{-1}}{1-0.927743 z^{-1}}
\end{array}\right]\left[\begin{array}{l}
u_{1}(k) \\
u_{2}(k)
\end{array}\right] .
\end{aligned}
$$

Applying the "ss" function in Matlab, we get the following 4-dimensional state-space model:

$$
\begin{aligned}
x(k+1)= & {\left[\begin{array}{cccc}
0.958 & 0 & 0 & 0 \\
0 & 0.9418 & 0 & 0 \\
0 & 0 & 0.9048 & 0 \\
0 & 0 & 0 & 0.9277
\end{array}\right] x(k) } \\
& +\left[\begin{array}{cc}
0.25 & 0 \\
0.25 & 0 \\
0 & 0.5 \\
0 & 0.5
\end{array}\right] u(k), \\
y(k)= & {\left[\begin{array}{cccc}
0.1678 & 0 & 0.9516 & 0 \\
0 & 0.2329 & 0 & 0.289
\end{array}\right] x(k) . }
\end{aligned}
$$

There are also constraints on the process inputs:

$$
\begin{array}{r}
-2 \leq u_{1} \leq 2, \quad-0.6 \leq u_{2} \leq 0.6 \\
-0.5 \leq \Delta u_{1} \leq 0.5, \quad 0.3 \leq \Delta u_{2} \leq 0.3 .
\end{array}
$$

The chosen MPCS parameters were $N=10, N_{u}=5$ and single scalar weighting $\boldsymbol{\Psi}=\mathbf{I}, \boldsymbol{\Lambda}=\lambda \mathbf{I}$, with $\lambda=0.1$.

The simulation scenario contained both step changes of the set-points (set-point trajectories are shown by dashed lines in the figures) and unmeasured input step disturbances $z(k)$, with step changes from zero to 0.3 and 
-0.2 , respectively, at sample $k=50$. The extended state observer for this case is of the form

$$
\begin{aligned}
& {\left[\begin{array}{c}
\hat{x}(k+1) \\
\hat{z}(k+1)
\end{array}\right]} \\
& =\left[\begin{array}{cc}
\mathbf{A} & \mathbf{B} \\
\mathbf{0} & \mathbf{I}
\end{array}\right]\left[\begin{array}{c}
\hat{x}(k) \\
\hat{z}(k)
\end{array}\right]+\left[\begin{array}{c}
\mathbf{B} \\
\mathbf{0}
\end{array}\right] u(k) \\
& \quad+\mathbf{K}_{\text {obs }}\left(y(k)-\left[\begin{array}{ll}
\mathbf{C} & \mathbf{0}
\end{array}\right]\left[\begin{array}{l}
\hat{x}(k) \\
\hat{z}(k)
\end{array}\right]\right),
\end{aligned}
$$

where the matrices $\mathbf{A}, \mathbf{B}, \mathbf{C}$ correspond to the process equations (45)- 46.

The poles of the process state observer (24) were chosen at $0.02,0.021,0.023,0.024$ and its initial point was [ $\left[\begin{array}{llll}1 & 1 & 1 & 1\end{array}\right]$, while the poles of the extended observer (47) were chosen at similar values, 0.02, 0.021, 0.023, $0.024,0.025,0.028$, with the initial point [ $\left[\begin{array}{llllll}1 & 1 & 1 & 1 & 0 & 0\end{array}\right]$. All simulations started with the system at a zero equilibrium point.

The results of simulations are shown in Figs. 13 , for cases with full state measurement, with the state observer (40) and with the extended observer (47), respectively. The gains of the extended observer turned out to be greater (about an order of magnitude), and therefore the values of the state estimates were larger and the corresponding output trajectories more variable, particularly during initial phase of damping the initial state estimation errors, but also after step changes of the input disturbances at $k=$ 50. Moreover, our tests showed an increased sensitivity of the extended observer to modeling errors.
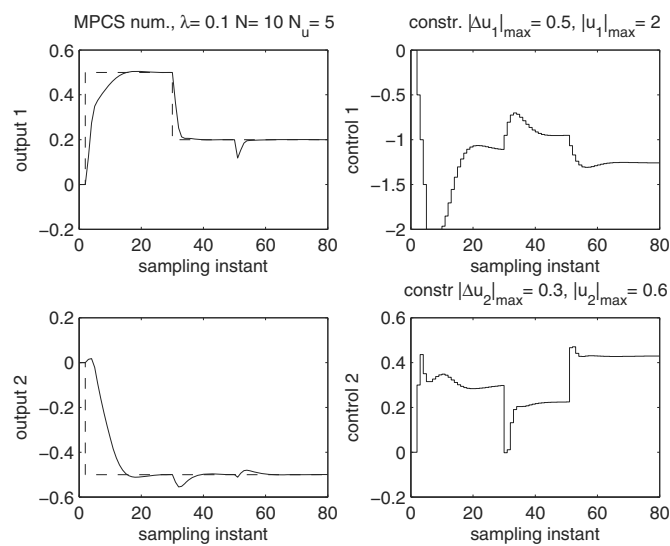

Fig. 1. MPCS with state measurement, under step changes of set-points (dashed line) and input disturbances (at $k=$ $50)$.

Additionally, trajectories of first two components of the state vector (solid lines) and the state estimate vector (dashed lines), corresponding to the trajectories from Fig. 2] are shown in Fig. 4. It can be seen that the observer (40) changes values of its estimates after $k=$ 50 , due to the influence of step changes of unmeasured
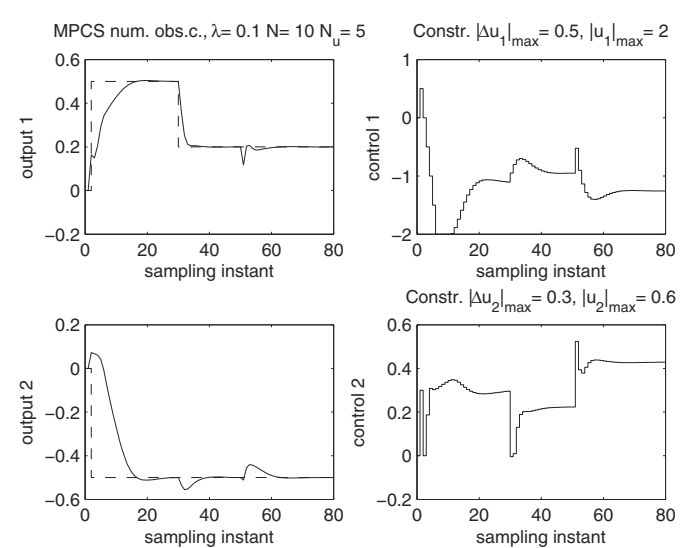

Fig. 2. MPCS with the state observer (40), under step changes of set-points (dashed line) and input disturbances (at $k=$ $50)$.
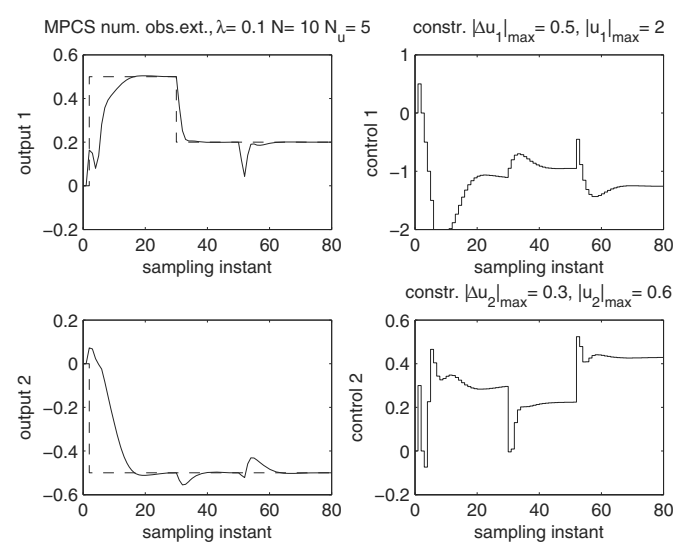

Fig. 3. MPCS with the extended state observer 477, under step changes of set-points (dashed line) and input disturbances (at $k=50$ ).
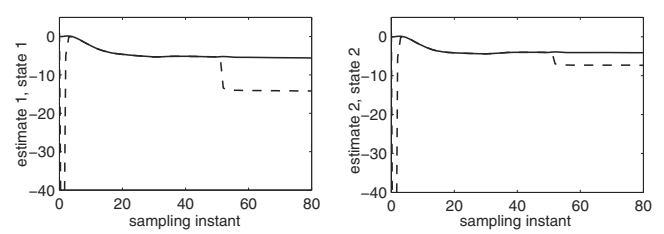

Fig. 4. Trajectories of the first two state components (solid lines) and observer outputs (dashed lines), corresponding to the trajectories from Fig. 2

input disturbances (and the estimation error is according to (30)).

Figure 5 presents the most demanding experiment: step changes (from zeros to 0.1 and -0.05 at sample $k=50$ ) of outer step disturbances affecting directly the process output were additionally added. Thus at sample $k=50$ four outer step disturbances are simultaneously 

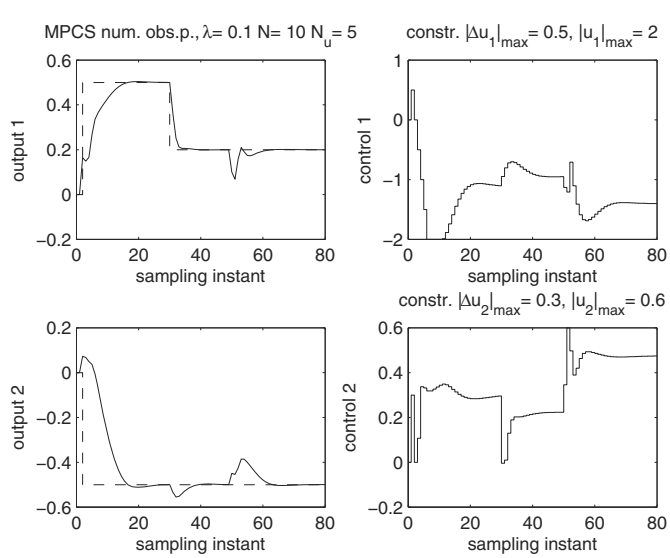

Fig. 5. MPCS with the state observer 40, under step changes of set-points (dashed line) and step changes of output and input disturbances (at $k=50$ ).

affecting the system: two input and two output ones. This is the case when the condition (38) is not satisfied and the extended dynamical system consisting of process and all disturbance states would be not detectable. The results shown in Fig. 5 clearly indicate that the proposed control structure works well in this case.

Finally, we added to the process state and output additional noises, $w_{x}(k)$ and $w_{y}(k)$, respectively, with normal distributions with zero mean and standard deviations 0.01 for all state variables and $(0.009,0.011)$ for the outputs, in addition to the unmeasured input disturbances, described earlier. Then the stationary Kalman filter for the process only (not extended) was designed (using the Matlab function dare), with matrices $\mathbf{Q}=10^{-3} \operatorname{diag}\left\{\begin{array}{llll}0.5 & 0.5 & 0.5 & 0.5\end{array}\right\}$ and $\mathbf{R}=$ $10^{-4} \operatorname{diag}\{0.20 .2\}$, which correspond to appropriately
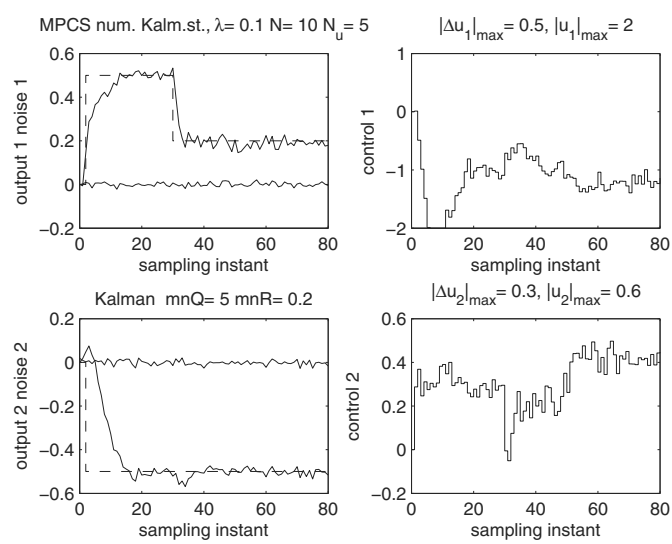

Fig. 6. MPCS with a stationary Kalman filter, under step changes of set-points (dashed line) and input disturbances, states and outputs with noise (shown also separately) added. tuned covariance matrices of the noise signals. The control system with this filter was tested-an example result is given in Fig. 6 It shows good performance of the proposed control structure with simplified state estimation, also under noisy conditions.

The presented example simulation results show that the proposed control system works correctly, confirming the theoretical analysis provided in this section. Based on these results, and others not presented here due to limited space (including also simulations with other processes), it can be concluded that the approach proposed in the paper should be recommended. It is simpler, using a less dimensional, standard observer of the process state, and more general, working also when a possibly restrictive condition concerning a maximal number of deterministic disturbances does not hold.

\section{MPCS with an extended, velocity form state-space model}

An alternative way to deal with deterministic disturbances, in order to assure offset-free control, is to use a specially reformulated model, with incremental state and corresponding state-space equations including output integrators (Prett and Garcia, 1988), which is called in the literature the velocity form model (see the works of Gonzalez et al. (2008), Maciejowski (2002) or Wang (2009) for applications to predictive control). In this approach, there is no need to compensate for deterministic disturbances, due to the incremental form of the model. The state-space equation is now defined as follows:

$$
\Delta x(k+1)=\mathbf{A} \Delta x(k)+\mathbf{B} \Delta u(k)
$$

where $\Delta x(k)=x(k)-x(k-1), \Delta u(k)=u(k)-u(k-1)$.

Defining the extended state vector as

$$
x_{r}(k)=\left[\Delta x(k)^{T} y(k)^{T}\right]^{T}
$$

and taking into account (48), we obtain

$$
\begin{aligned}
y(k+1)-y(k) & =\mathbf{C} \Delta x(k+1) \\
& =\mathbf{C A} \Delta x(k)+\mathbf{C B} \Delta u(k) .
\end{aligned}
$$

Thus, we get the following extended state-space equations:

$$
\begin{aligned}
{\left[\begin{array}{c}
\Delta x(k+1) \\
y(k+1)
\end{array}\right]=} & {\left[\begin{array}{cc}
\mathbf{A} & \mathbf{0} \\
\mathbf{C A} & \mathbf{I}
\end{array}\right]\left[\begin{array}{c}
\Delta x(k) \\
y(k)
\end{array}\right] } \\
& +\left[\begin{array}{c}
\mathbf{B} \\
\mathbf{C B}
\end{array}\right] \Delta u(k), \\
y(k)= & {\left[\begin{array}{ll}
\mathbf{0} & \mathbf{I}
\end{array}\right]\left[\begin{array}{c}
\Delta x(k) \\
y(k)
\end{array}\right] . }
\end{aligned}
$$


Introducing individual symbols for matrices in 51 and 52), we can write these equations in compact form,

$$
\begin{aligned}
x_{r}(k+1) & =\mathbf{A}_{r} x_{r}(k)+\mathbf{B}_{r} \Delta u(k), \\
y(k) & =\mathbf{C}_{r} x_{r}(k) .
\end{aligned}
$$

We shall denote the described algorithm by the acronym MPCSE (MPC with a State-space Extended model).

The advantage of the state model (51) is that using the difference form of state and control signals eliminates constant (step) disturbances and removes integration from the integrated white noise. Therefore, introduction of state disturbance $v(k)$ in our approach, or introduction and estimation of disturbances $z(k)$ in the conventional approach with (36) and (37), is no longer needed. Moreover, the second (lower) vector equation from (51) describes discrete-time integration of the output $y(k)$, thus attenuating process output disturbances. Therefore, the control will be offset-free for all deterministic disturbances and integrated white noise. Consequently, the disturbances do not enter the predictions, the predicted free output trajectory takes the form (cf. (15))

$$
Y^{0}(k)=\widetilde{\mathbf{C}_{r}} \widetilde{\mathbf{A}_{r}} x_{r}(k),
$$

where matrices $\widetilde{\mathbf{C}_{r}}, \widetilde{\mathbf{A}_{r}}$ are defined by (17) and (18), provided matrices $\mathbf{C}, \mathbf{A}$ are replaced there by $\mathbf{C}_{r}, \mathbf{A}_{r}$.

However, there is a cost to be paid for the advantage: it follows directly from the structure of the extended state matrix $\mathbf{A}_{r}$, see (51), that it retains eigenvalues of $\mathbf{A}$ and adds $n_{y}=\operatorname{dim} y$ eigenvalues equal to 1 . Thus, numerical properties of the predictions and of the optimization problem (QP) get worse and may cause numerical troubles, especially for longer prediction horizons.

A standard approach is to design an observer of the extended state (see the tutorial paper by Gonzalez et al. (2008)). However, the observer designed for estimation of the process state $x(k)$ only, despite disturbances, as proposed earlier in this paper, can also be applied here. Certainly, the disturbances will also bias the state estimates, similarly as was discussed in the previous section. As the process outputs are always measured, the proposed procedure is as follows:

1. an observer of the original process state $x(k)$ is designed,

2. an estimate of the extended state is computed based on the last two original state estimates and the output measurement,

$$
\hat{x}_{r}(k)=\left[(\hat{x}(k)-\hat{x}(k-1))^{T} y(k)^{T}\right]^{T} .
$$

Under constant state disturbances (which includes input disturbances), the process state estimate $\hat{x}(k)$ will be biased by an offset, but this error will be eliminated in the extended state estimate $\hat{x}_{r}(k)$, due to subtraction of neighboring state estimates in 56 .
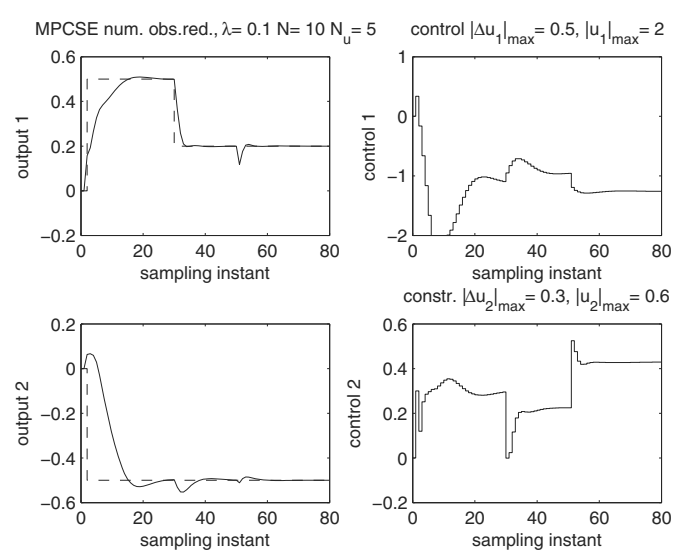

Fig. 7. MPCSE with an observer of process state $x(k)$, with step changes of set-points and input disturbances (at $k=50$ ).

Example 2. We shall now present results of simulations for the process introduced earlier in Example 1, but now with the MPCSE controller, with a standard current observer applied to the process state $x(k)$ and the extended state estimate calculated according to (56). The initial values of the process state and of the observer state are as defined previously. The simulation scenario is also the same as used in Example 1 before, with step changes of set-points and input disturbances. A representative result is presented in Fig. 7 showing proper operation of the proposed control scheme.

The results presented in Figs. 2 and 7 are very similar, there were also no problems with numerical solution of the QP problem for the case with the velocity form state-space model and computation times were similar for both the cases. This is due to the fact that our test problem is rather simple, and relatively short prediction and control horizons were sufficient. Moreover, in both cases the same process state observer is used in the proposed MPC control scheme. Therefore, it could be concluded that, for relatively simple control problems, the choice is a matter of taste. For more complex cases, with more difficult dynamics and longer horizons, the comparison needs appropriate research. It was not the aim of this paper.

\section{Conclusions}

The paper addressed problems of proper handling of deterministic constant-type disturbances and design of state estimators, for the use in constrained MPC controllers with linear state-space process models. A systematic discussion was provided, first for the case with a full measured state and then for the case with an unmeasured state and state estimation. It was recalled that using constant state disturbance prediction in the case with a measured state enables offset-free control without the 
need for observer design. For the case with unmeasured state (with a measured process output only) a new MPC control structure was proposed, with the observer of a pure process state only, working properly due to application of constant state disturbance prediction and an appropriate correction term in the process output prediction equations.

The proposed control structure is competitive compared with the conventional one with extended process-and-disturbance state modeling and an extended state observer. It is simpler, easier to design and applicable to a wider class of problems, as detectability conditions of an extended process-and-disturbance state model are more restrictive. An extension to Kalman filtering is also straightforward. Application of the proposed approach to alternative process modeling with extended state in complete velocity form was also proposed. The results were illustrated on several representative simulations of a MIMO $(2 \times 2)$ process model representing a linearized continuous flow reactor model.

\section{Acknowledgment}

The author is grateful to reviewers for their comments and encouragement to improve the final version of the paper. This work is a revised, extended version of the paper presented at the 18th IFAC World Congress (Milano, Italy, 2011).

\section{References}

Anderson, D. and Moore, J. (2005). Optimal Filtering, Dover Publications Inc, New York, NY.

Astrom, K. and Wittenmark, B. (1997). Computer Controlled Systems, Prentice Hall, Upper Saddle River, NJ.

Blevins, T.L., McMillan, G.K., Wojsznis, W.K. and Brown, M.W. (2003). Advanced Control Unleashed, The ISA Society, Research Triangle Park, NC.

Blevins, T.L., Wojsznis, W.K. and Nixon, M. (2013). Advanced Control Foundation, The ISA Society, Research Triangle Park, NC.

Camacho, E. and Bordons, C. (1999). Model Predictive Control, Springer Verlag, London.

Doyle III, F., Ogunnaike, B. and Pearson, R. (1996). Nonlinear model predictive control of a simulated multivariable polymerization reactor using second-order Volterra models, Automatica 32(9): 1285-1301.

Gonzalez, A.H., Adam, E.J. and Marchetti, J.L. (2008). Conditions for offset elimination in state space receding horizon controllers: A tutorial analysis, Chemical Engineering and Processing 47(12): 2184-2194.

Hesketh, T. (1982). State-space pole-placing self-tuning regulator using input-output values, IEE Proceedings, Part D 129(4): 123-128.
Ławryńczuk, M. (2009). Efficient nonlinear predictive control based on structured neural models, International Journal of Applied Mathematics and Computer Science 19(2): 233-246, DOI: 10.2478/v10006-009-0019-1.

Ławryńczuk, M. and Tatjewski, P. (2010). Nonlinear predictive control based on neural multi-models, International Journal of Applied Mathematics and Computer Science 20(1): 7-21, DOI: 10.2478/v10006-010-0001-y.

Maciejowski, J. (2002). Predictive Control, Prentice Hall, Harlow.

Maeder, U. and Morari, M. (2010). Offset-free reference tracking with model predictive control, Automatica 46(9): 1469-1476.

Morari, M. and Maeder, U. (2012). Nonlinear offset-free model predictive control, Automatica 48(9): 2059-2067.

Muske, K. and Badgwell, T. (2002). Disturbance modeling for offset-free linear model predictive control, Journal of Process Control 12(5): 617-632.

Pannocchia, G. and Bemporad, A. (2007). Combined design of disturbance model and observer for offset-free model predictive control, IEEE Transactions on Automatic Control 52(6): 1048-1053.

Pannocchia, G. and Rawlings, J. (2003). Disturbance models for offset-free model predictive control, AIChE Journal 49(2): 426-437.

Prett, D. and Garcia, C. (1988). Fundamental Process Control, Butterworths, Boston, MA

Qin, S. and Badgwell, T. (2003). A survey of industrial model predictive control technology, Control Engineering Practice 11(7): 733-764.

Rao, V. and Rawlings, J.B. (2009). Model Predictive Control: Theory and Design, Nob Hill Publishing, Madison, WI.

Rossiter, J. (2003). Model-Based Predictive Control, CRC Press, Boca Raton, FL.

Tatjewski, P. (2007). Advanced Control of Industrial Processes, Springer Verlag, London.

Tatjewski, P. (2008). Advanced control and on-line process optimization in multilayer structures, Annual Reviews in Control 32(1): 71-85.

Tatjewski, P. (2010). Supervisory predictive control and on-line set-point optimization, International Journal of Applied Mathematics and Computer Science 20(3): 483-495, DOI: 10.2478/v10006-010-0035-1.

Tatjewski, P. (2011). Disturbance modeling and state estimation for predictive control with different state-space process models, Preprints of the 18th IFAC World Congress, Milan, Italy, pp. 5326-5331.

Tatjewski, P. (2012). Modeling deterministic disturbances and state filtering in model predictive control with state-space models, in M. Busłowicz and K. Malinowski (Eds.), Advances in Control Theory and Automation, OWPB, Białystok, pp. 263-274.

Tatjewski, P. and Ławryńczuk, M. (2006). Soft computing in model-based predictive control, International Journal of Applied Mathematics and Computer Science 16(1): 7-26. 
Wang, L. (2009). Model Predictive Control System Design and Implementation Using MATLAB, Springer Verlag, London.

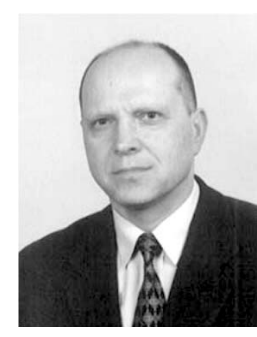

Piotr Tatjewski, M.Sc. (1972), Ph.D. (1976),

D.Sc. (1988) in control engineering from the Warsaw University of Technology (WUT). Since 1972 with the Institute of Control and Computation Engineering (ICCE) at the WUT Faculty of Electronics and Information Technology. Since 1993 a professor, since 2006 tenured, 19962008: the director of the ICCE, currently the head of the Control and Software Engineering Division and the faculty vice-dean for research. Member of the EUCA (European Union Control Association), the Administrative Council (2007-2011), the Committee on Automatics and Robotics of the Polish Academy of Sciences. Author of 6 books ( 3 in English), 16 book chapters, over 40 journal papers and 75 published conference papers. Leader of several research projects, including direct cooperation with industry. Main scientific interests: advanced control, linear and nonlinear predictive control, multilayer control and on-line set-point optimization, soft computing techniques in control, hierarchical methods in optimization and control.

Received: 27 September 2013

Revised: 8 January 2014 